\title{
Microresonator Brillouin gyroscope
}

\author{
Jiang LI, ${ }^{1,2}$ Myoung-Gyun Suh, ${ }^{1}$ and Kerry Vahala ${ }^{1, *}$ \\ ${ }^{1} T$. J. Watson Laboratory of Applied Physics, California Institute of Technology, Pasadena, California 91125, USA \\ ${ }^{2}$ Present address: hQphotonics Inc., 2500 E. Colorado Blvd., Pasadena, California 91107, USA \\ *Corresponding author: vahala@caltech.edu
}

Received 14 November 2016; revised 6 February 2017; accepted 9 February 2017 (Doc. ID 280805); published 7 March 2017

\begin{abstract}
Optical-based rotation sensors have revolutionized precision, high-sensitivity inertial navigation systems. At the same time these sensors use bulky optical fiber spools or free-space resonators. A chip-based, micro-optical gyroscope is demonstrated that uses counterpropagating Brillouin lasers to measure rotation as a Sagnac-induced frequency shift. Preliminary work has demonstrated a rotation-rate measurement that surpasses prior micro-optical rotation-sensing systems by over 40-fold. ๑2017 Optical Society of America
\end{abstract}

OCIS codes: (190.5890) Scattering, stimulated; (140.3370) Laser gyroscopes; (130.0130) Integrated optics.

https://doi.org/10.1364/OPTICA.4.000346

Inertial sensors for rotation are used widely in commercial and military systems. Driven by applications in consumer electronics and miniature satellites, there has been intense interest in compact, lightweight sensors that can be integrated with electronics. MEMS-based sensors have captured this miniature lightweight application space, but do not provide sensitivity and bias stability that competes with ring laser gyros [1] and fiber-optic gyros [2]. Moreover, due to their mechanical nature, MEMS-based devices are less immune to shock and vibration [3]. The prospect of applying micro-fabrication methods to optical-based gyro systems is therefore appealing, both because it addresses environmental concerns (even beyond conventional optical gyro systems) and it might offer performance that exceeds MEMS-based systems. A new class of micro-optical gyro is demonstrated that uses counterpropagating Brillouin lasers in a chip-based optical resonator to measure rotation as a frequency shift [4]. Preliminary work has measured sinusoidal rotations with rates as low as $22 \mathrm{deg} / \mathrm{h}$, which surpasses prior experimental micro-optical systems by over 40-fold [5,6].

A Brillouin laser ring gyro (BLRG) was studied in the 1990s using an optical fiber resonator [7]. Brillouin scattering in silica optical fiber results from the interaction of an optical pump wave with microwave-rate phonons [8]. At sufficiently high pump intensities, the process amplifies counterpropagating waves residing within a narrow frequency band (about $50 \mathrm{MHz}$ wide) that is downshifted relative to the pump frequency by $10-11 \mathrm{GHz}$ (for pumping near $1.55 \mu \mathrm{m}$ ). In a resonator, this amplification can overcome round-trip losses to produce a lasing Stokes wave. In the BLRG, a fiber ring resonator was bidirectionally pumped to excite two counterpropagating Brillouin lasers [7]. Upon rotation of the resonator, these counterpropagating lasers experienced opposing frequency shifts caused by the Sagnac effect [9] Heterodyne detection of the two laser signals allowed measurement of the rotation rate. As in a conventional ring laser gyro, the counterpropagating laser lines produce a narrow-linewidth beatnote on account of laser action in a high- $Q$ cavity. Co-lasing within a common resonator also tends to cancel technical noise contributions to the beatnote linewidth.

In this work, the Brillouin laser cavity is a high-optical- $Q$ micro resonator that measures only $18 \mathrm{~mm}$ in diameter and is fabricated of silica on a silicon chip [10]. Also, the method of excitation relies on a single pump wave to induce a Brillouin laser cascade [11]. In this approach, a first Stokes wave is generated as before. However, upon further pumping this Stokes wave increases in power to a point where it will act to pump a second Brillouin Stokes wave, propagating in a direction opposite to the first Stokes wave. This cascade process can be made to continue for many Stokes orders, each time producing a Stokes laser line that is shifted to a lower optical frequency and counterpropagating relative to the previous line. Neighboring Stokes waves produced in this cascaded fashion will experience opposing Sagnac shifts and are well-suited for the gyro application. Moreover, because the neighboring Stokes waves are distinct cavity modes separated in frequency by over $10 \mathrm{GHz}$, the lock-in effect commonly present in laser gyroscopes is not present. The free-spectral -range (FSR) of the resonator is designed to precisely match an integer fraction of the Brillouin shift (1/3 in the present case). The counterpropagating even- and odd-order Stokes waves are combined on a fast photodetector to generate a microwave beat note with subHertz linewidth $[11,12]$. This beatnote is electrically processed to provide the rotation readout [see Fig. 1(a)].

A detailed schematic of our experimental setup is shown in Fig. 1(b). The upper block contains the gyro sensing unit, which at its core includes the high- $Q$ disk microresonator and fast photodetector. The optical pump laser (external cavity diode laser) is evanescently coupled to the high- $Q$ disk resonator using a tapered fiber. The cavity mode used for stimulated Brillioun scattering (SBS) generation has a loaded $Q$ of 126 million. The pump laser is locked to the cavity resonance using the Pound-Drever-Hall technique. The pump threshold for the first Brillouin Stokes wave is about $250 \mu \mathrm{W}$. Cascaded Brillouin Stokes laser action is generated to the third order when the pump power is increased to a few milliwatts. The pump wave and 

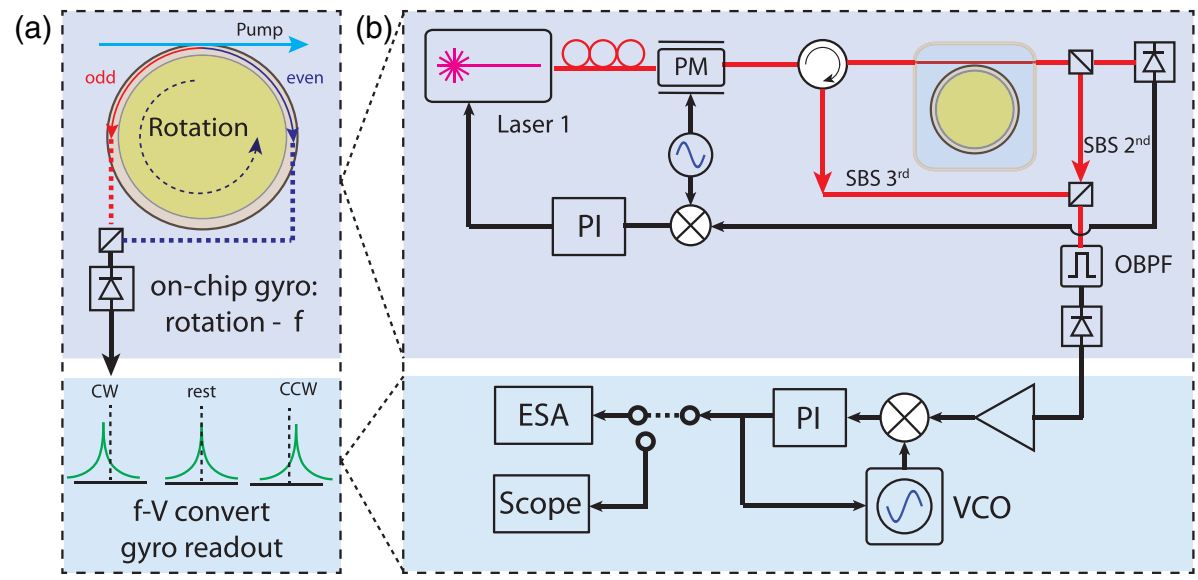

Fig. 1. (a) Simplified schematic illustrating the principle of Brillouin laser gyroscope operation. Optical pumping (clockwise direction) induces Brillouin laser action, which results in cascaded odd (counter-clockwise, CCW) and even (clockwise, CW) order Stokes lasers. These lasing modes experience opposing Sagnac frequency shifts. Detection of the beat frequency of these co-lasing signals followed by a frequency-to-voltage readout $(\mathrm{f}-\mathrm{V})$ provides rotation sensing. Laser action on odd Stokes (red) and even Stokes (blue) lines is shown. CW, rest, and CCW rotation induces beat frequency shifts as indicated. (b) Upper panel is a detailed experimental schematic for the gyroscope. The microcavity (yellow) is optically pumped. The laser pump is locked to a microcavity resonance using a Pound-Drever-Hall lock. Second-order and third-order Stokes co-lasing optical signals are coupled onto a photodetector using a circulator and bidirectional coupler. The laser lines are filtered using an optical band-pass filter (OBPF) before detection. In the lower panel, the frequency-to-voltage readout system is shown. A voltage-controlled oscillator (VCO) is phase locked to the detected beat frequency and the servo output provides a calibrated frequency readout. The readout is analyzed using an oscilloscope (Scope) and an electrical spectrum analyzer (ESA). Also shown in the figure: PI, proportional integral servo; PM, phase modulator.

second-order Stokes wave propagate along the clockwise $(\mathrm{CW})$ direction and are coupled out of the resonator in the forward direction, while the first and the third Stokes waves propagate along the counterclockwise (CCW) direction and are coupled out of the resonator in the backward direction. A fiber circulator is used to route the backward-propagating waves. The second and third Stokes waves are selected by a narrow-bandwidth optical bandpass filter (OBPF). Finally, a fast photodetector is used to detect the microwave beat note at $10.872 \mathrm{GHz}$ between the second and the third Stokes waves.

The lower block in Fig. 1(b) creates the gyro readout. It is a frequency-to-voltage converter consisting of a voltage-controlled oscillator (VCO) locked to the Brillouin beatnote. The VCO is compared against the Brillouin beatnote using a mixer and the phase error signal from the mixer locks the VCO using a proportional-integral (PI) servo. Because the PI servo output signal is applied to the VCO tuning port with known tuning coefficient (here at $10 \mathrm{kHz} / \mathrm{V}$ ), the servo output signal is proportional to the frequency change of the Brillouin beat note. Therefore, the servo output signal can be used as the gyro readout signal and analyzed on an electrical spectrum analyzer (ESA) or an oscilloscope.

To test the gyro, a sinusoidal rotation with angular amplitude of $0.14^{\circ}$ is applied to the Brillouin gyro. The rotation oscillates at $7.5 \mathrm{~Hz}$. For the measurement, the resonator was packaged into a small box with pigtailed connectors, as shown in Fig. 2(a). To create the sinusoidal rotation, one corner of the box was hinged while the other end was moved using a piezoelectric pusher. Diagrams of the resonator being rotated are presented with the corresponding gyro readout signal (servo output signal) and the angular displacement signal (as acquired on an oscilloscope) in Figs. 2(b) -2 (d). There is a $\pi / 2$ phase shift between the angular displacement signal and the gyro readout signal (SBS beat note change) since the beatnote frequency change is proportional to the angular velocity (derivative of angular displacement).
Moreover, the sign of the Brillouin beat note change indicates the direction of rotation. In the measured configuration, a $\mathrm{CW}$ rotation [indicated in Fig. 2(b) and the blue-stripe regions in Fig. 2(d)] decreases the second Stokes wave frequency $\left(\nu_{2}\right)$ and increases the third Stokes wave frequency $\left(\nu_{3}\right)$. Therefore, a CW rotation induces a negative shift in the Brillouin beatnote frequency $\left(\nu_{2}-\nu_{3}\right)$. On the other hand, a CCW rotation [indicated in Fig. 2(c) and the red-stripe regions in Fig. 2(d)] will induce a positive shift in the Brillouin beatnote frequency.

(a)
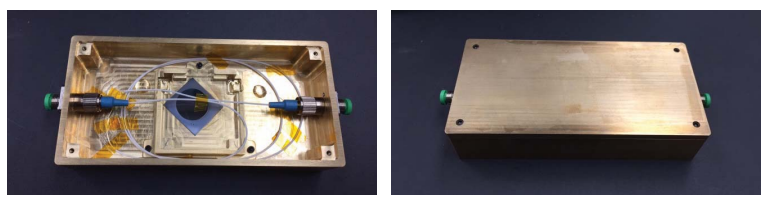

(b)

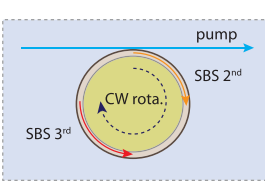

(c)

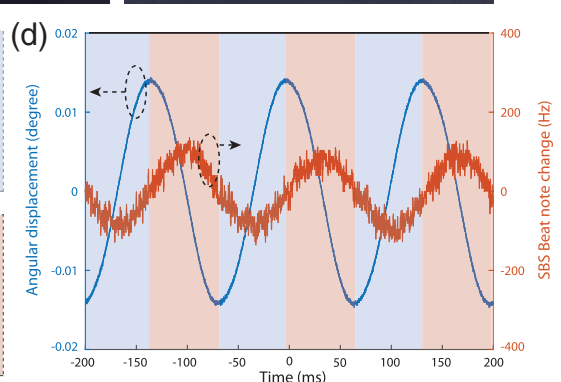

Fig. 2. (a) A gyro resonator was packaged into a fiber-connectorized box for rotation measurement. Left panel shows the box with the lid removed, and the $18 \mathrm{~mm}$ diameter resonator is visible as the gray silicon chip. (b), (c) CW rotation and CCW rotation of the SBS gyroscope with second- and third-order Stokes laser signals as indicated. Background coloring of the two rotation cases presented in (b) and (c) corresponds to the shaded regions in (d). (d) Time domain measurement of gyroscope output under sinusoidal rotation. Blue, angular displacement applied to the gyroscope; red, measured Sagnac frequency shift. 
(a)

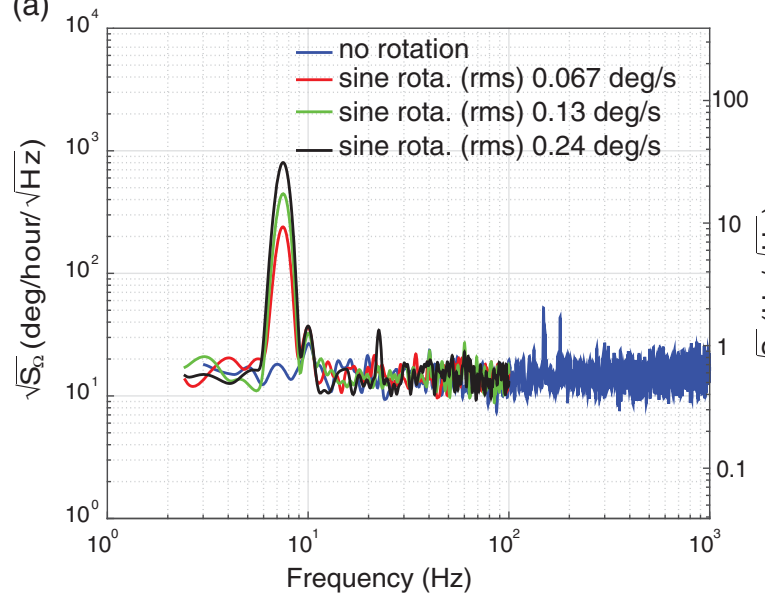

(b)

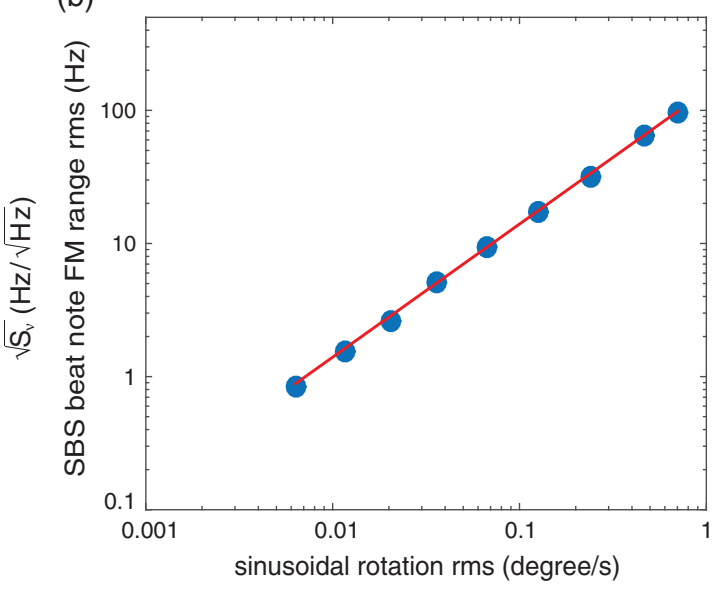

Fig. 3. (a) Measurement of sinusoidal rotations using an electrical spectrum analyzer. The rms rotation rates are given in the legend. Left scale gives the gyroscope sensitivity as set by the white noise floor. An alternate scale giving the frequency-noise spectral density is provided on the right axis. (b) Plot of rms Sagnac frequency shift versus rms angular rotation rate measured as shown in (a).

When the gyroscope is at rest, the frequency noise of the Brillouin beat note within the VCO locking bandwidth determines the rotation sensitivity of the gyroscope. This frequency noise can be measured from the servo output signal using the ESA. According to the Sagnac effect, the rotation sensitivity $\left(\sqrt{S_{\delta \Omega}}\right)$ of the gyroscope is related to the frequency noise $\left(\sqrt{S_{\delta \nu}}\right)$ of the beatnote between the two counterpropagating Brillouin lasers: $\sqrt{S_{\delta \Omega}}=\frac{n \lambda}{D} \sqrt{S_{\delta \nu}}$, where $n$ is the refractive index of the cavity medium, $\lambda$ is the laser wavelength in vacuum, and $\mathrm{D}$ is the diameter of the microresonator. The blue noise spectrum in Fig. 3(a) shows the measured rotation sensitivity of the gyroscope at rest. A white frequency noise of about $0.6 \mathrm{~Hz} / \sqrt{\mathrm{Hz}}$ (right axis) is obtained from $3 \mathrm{~Hz}$ to $1 \mathrm{kHz}$ offset, which corresponds to a rotation sensitivity of about $15 \mathrm{deg} / \mathrm{h} / \sqrt{\mathrm{Hz}}$ (left axis) from $3 \mathrm{~Hz}$ to $1 \mathrm{kHz}$ bandwidth.

When the gyroscope undergoes a sinusoidal rotation [as shown in Fig. (2)], the output signal also shows a sinusoidal modulation. Figure 3(a) shows the electrical spectrum of the gyroscope readout when the gyroscope undergoes a sine rotation at $7.5 \mathrm{~Hz}$ with the following rms rotation rates: $0.24 \mathrm{deg} / \mathrm{s}$ (black curve), $0.13 \mathrm{deg} / \mathrm{s}$ (green curve), and $0.067 \mathrm{deg} / \mathrm{s}$ (red curve). A corresponding change in the modulation tone amplitude at $7.5 \mathrm{~Hz}$ in the spectrum can be seen. The resolution bandwidth in the measurement is $1 \mathrm{~Hz}$. In Fig. 3(b), the blue circles give a series of rms Sagnac frequencies (recorded from the ESA) plotted versus the rms angular rotation rate at the $7.5 \mathrm{~Hz}$ modulation rate. The red line is calculated from the Sagnac formula and agrees with the measurement (Sagnac formula: $\delta \Omega=(n \lambda / D) \delta \nu$, where $\delta \Omega$ is the rotation rate and $\delta \nu$ is the frequency shift of the Brillouin beat note). Moreover, a minimum rms rotation rate value of $6.3 \times$ $10^{-3} \mathrm{deg} / \mathrm{s}$ (or $22 \mathrm{deg} / \mathrm{h}$ ) is detected. Compared with the previous reported sensitivity of a passive micro-optical gyro (900 deg/h, and gyro bandwidth of $9 \mathrm{~Hz}$ ) [5], this represents a 40 -fold improvement of minimum rotation rate detection, and with more than $1 \mathrm{kHz}$ of gyro bandwidth.

In conclusion, an on-chip Brillouin gyroscope has been demonstrated with a sensitivity of $15 \mathrm{deg} / \mathrm{h} / \sqrt{\mathrm{Hz}}$ and with detection bandwidth of more than $1 \mathrm{kHz}$. A minimum rotation rate of $22 \mathrm{deg} / \mathrm{h}$ was measured, which represents a 40 -fold improvement over previous micro-resonant gyroscopes. Improvements directed to the linewidth of the Brillouin lasers will directly improve the gyro sensitivity. For example, a 10-fold improvement in linewidth would place the sensitivity in the range of a few degrees/hour. The $\mathrm{X}$-band-rate beat frequency produced by the counterpropagating Brillouin waves presents challenges with respect to gyroscope bias stability and drift [13]. Current efforts are focused on addressing these environmental-drift-related issues. With future improvements this gyroscope might provide complementary performance to conventional bulk ring laser gyros and fiber-optic gyroscopes by offering miniature size, light weight, and low power consumption with improved resistance to shock and vibration.

During the preparation of this paper, a conference proceeding by Maleki et al. on a resonant optical micro-gyroscope was reported [14].

Funding. Defense Advanced Research Projects Agency (DARPA) (DARPA PRIGM:AIMS program); Kavli Nanoscience Institute.

Acknowledgment. The authors would like to thank Seung Hoon Lee for helpful discussions.

\section{REFERENCES}

1. W. Chow, J. Gea-Banacloche, L. Pedrotti, V. Sanders, W. Schleich, and M. Scully, Rev. Mod. Phys. 57, 61 (1985).

2. H. C. Lefevre, The Fiber-Optic Gyroscope (Artech House, 2014).

3. K. Liu, W. Zhang, W. Chen, K. Li, F. Dai, F. Cui, X. Wu, G. Ma, and Q. Xiao, J. Micromech. Microeng. 19, 113001 (2009).

4. J. Li, M.-G. Suh, and K. Vahala, in Nonlinear Optics Conference, OSA Technical Digest (online) (Optical Society of America, 2015), paper NTh3A.2.

5. H. Ma, W. Wang, Y. Ren, and Z. Jin, IEEE Photon. Technol. Lett. 25, 198 (2013).

6. F. Dell'Olio, T. Tatoli, C. Ciminelli, and M. Armenise, J. Eur. Opt. Soc. Rapid Pub. 9, 14013 (2014).

7. F. Zarinetchi, S. Smith, and S. Ezekiel, Opt. Lett. 16, 229 (1991).

8. E. Ippen and R. Stolen, Appl. Phys. Lett. 21, 539 (1972).

9. G. Sagnac, C. R. Acad. Sci. 157, 708 (1913).

10. H. Lee, T. Chen, J. Li, K. Y. Yang, S. Jeon, O. Painter, and K. J. Vahala, Nat. Photonics 6, 369 (2012).

11. J. Li, H. Lee, T. Chen, and K. J. Vahala, Opt. Express 20, 20170 (2012).

12. J. Li, H. Lee, and K. J. Vahala, Nat. Commun. 4, 2097 (2013).

13. H. Ma, J. Zhang, L. Wang, and Z. Jin, Opt. Express 23, 15088 (2015).

14. L. Maleki, W. Liang, D. Eliyahu, V. Ilchenko, A. Savchenkov, and A. Matsko, in 2016 IEEE Photonics Conference Technical Digest (online) (IEEE, 2016), paper TuD1.3. 